\title{
La migration régionale et entrepreneuriale des Libanais de Dakar, Abidjan et Ouagadougou
}

\begin{abstract}
Marwa El Chab ${ }^{1}$
[Résumé] A contrario de l'analyse communautariste concernant la présence libanaise en Afrique de l'Ouest, nous proposons une grille de lecture qui prend en compte la mobilité régionale des Libanais en fonction des conditions sociopolitiques et des opportunités économiques. Sur la base d'une recherche doctorale en anthropologie sociale menée à Dakar, Abidjan et Ouagadougou, nous postulons l'existence de deux types de migrations régionales - l'une axée sur l'initiative personnelle, et l'autre sur l'initiative d'entreprises qui recrutent leurs cadres en fonction de leur appartenance nationale. Cette perception amène à réinterroger les concepts de « diaspora » et de middleman minorities classiquement mobilisés à propos de la présence libanaise en Afrique de l'Ouest mais entretenant des zones d'ombres, notamment autour de l'extraterritorialité des «communautés » et de leur mobilisation stratégique des nationalités africaines.

Mots-clés : Middleman minorities, diaspora, extraterritorialité, nationalité, Libanais, Afrique de l'Ouest.

[Abstract] In contrast to the communitarian analysis of the Lebanese presence in West Africa, we suggest an analytical framework that takes into account Lebanese regional mobility based on socio-political conditions and economic opportunities. During our doctoral research in social anthropology, we conducted fieldwork in Dakar, Abidjan and Ouagadougou and we observed the existence of two types of regional migration - one related to individual initiative and another one based on the initiative of companies which recruit their managers according to their national belongings. This perception leads to re-examine the concepts of "diaspora" and "middleman minorities" which are traditionally mobilized in the study of Lebanese people in West Africa but maintain a grey zone, especially about the extraterritoriality of "communities" and their strategic mobilization of African nationalities.

Keywords: Middleman minorities, diaspora, extraterritoriality, nationalities, Lebanese,
\end{abstract} West Africa.

\section{Introduction}

Les études sur les Libanais en Afrique ont souvent passé sous silence le phénomène de la mobilité régionale de cette population en Afrique de l'Ouest. La présence libanaise dans cette région date pourtant du début du $\mathrm{XX}^{\mathrm{e}}$ siècle. Elle est le plus souvent analy-

1 École des hautes études en sciences sociales, Paris, France. 
sée comme une migration du pays d'origine, le Liban, vers les pays d'accueil africains (Binet, 1975; Taan, 1988).

Nous défendons ici la nécessité de considérer l'histoire de la population libanaise en Afrique de l'Ouest comme partie intégrante de l'histoire de la région Ouest-africaine 2 . À partir de l'étude de la mobilité des entrepreneurs libanais en Afrique de l'Ouest, nous souhaitons révéler la complexité des dynamiques socio-économiques africaines. Nous appuyons notre argumentaire sur la littérature ayant trait aux économies africaines et aux Libanais en Afrique de l'Ouest ainsi que sur une enquête issue d'une thèse en anthropologie sociale, menée à Dakar au Sénégal, à Abidjan en Côte d'Ivoire et à Ouagadougou au Burkina Faso, entre juillet 2012 et mai 2014. Cette enquête a donné lieu à des observations ethnographiques et à la réalisation de cent trente-sept entretiens. Nous nous appuierons ici spécifiquement sur dix-sept entretiens menés à Ouagadougou auprès d'interviewés ayant effectué un déplacement, pour des raisons professionnelles, de Dakar vers Ouagadougou, parfois via Abidjan, afin de mettre en évidence la mobilité régionale des enquêtés. Nos matériaux conduisent également à distinguer deux comportements migratoires à l'œuvre dans la région : le premier relève d'initiatives personnelles et mobilise des réseaux de connaissances familiales et interpersonnelles; le second répond aux besoins d'expansion d'entreprises recrutant les cadres de leurs succursales ouest-africaines dans le pays où est situé leur siège.

Enfin, les choix théoriques qui dominent dans les études sur l'entrepreneuriat libanais en Afrique de l'Ouest peuvent, à la lumière de ces données, être questionnés. C'est particulièrement le cas en ce qui concerne l'usage des concepts de "diaspora », de middleman minorities, d'extraterritorialité des «communautés » libanaises d'Afrique et de «mobilisation stratégique » de nationalités par les individus. À partir d'une synthèse de la littérature scientifique concernant les Libanais en Afrique de l'Ouest, nous pointerons les limites des concepts et les angles morts de la littérature. Nous présenterons ensuite une lecture descriptive des données issues du terrain ouagalais puis proposerons, pour finir, une grille de lecture de la mobilité régionale des Libanais intégrant les conditions sociopolitiques et les opportunités économiques présentes dans la région.

\section{Les Libanais d'Afrique de l'Ouest dans la littérature}

Les premières études menées sur les Libanais en Afrique de l'Ouest sont teintées de problématiques coloniales (Desbordes, 1938)3. Les catégorisations souvent stigmati-

2 Akyeampong va plus loin en suggérant que la place des Libanais dans les structures sociales africaines - notamment dans le cas du Ghana qu'il examine - trouve sa raison dans la colonisation (Akyeampong, $2006: 300$ ).

3 Dans Faire fortune en Afrique, Benjamin Rubbers souligne : « Les Indiens en Afrique de l'Est et les Libanais en Afrique de l'Ouest attirent l'attention des historiens, des sociologues et des anthropologues au tournant des années 1960. Mais ils disparaissent ensuite de l'agenda des 
santes dont elles témoignent sont, pour certaines d'entre elles, toujours mobilisées aujourd'hui. La présence libanaise a, depuis le début des années 1900, provoqué des controverses. L'analyse des archives disponibles indique en effet une opinion coloniale scindée en deux : d'une part, une politique de l'Empire encourageant l'installation des Libanais en Afrique de l'Ouest ; de l'autre, une administration coloniale travaillant activement à limiter leur immigration. Ces tensions reflètent les inquiétudes des petits entrepreneurs européens face à l'«invasion libanaise » ou aux pratiques commerçantes « déloyales » des Libanais.

\subsection{La narration relative aux origines de cette migration}

La migration libanaise vers l'Afrique de l'Ouest est indissociable de l'émigration des populations levantines de l'Empire ottoman à la fin du XIX ${ }^{\mathrm{e}}$ et au début du XX $\mathrm{XX}^{\mathrm{e}}$ siècle. Les premiers migrants originaires du mont Liban arrivèrent à cette période. Ces flux se poursuivirent lors de la constitution de l'entité politique libanaise sous mandat français en 1920. Ils perdurent jusqu'à nos jours, avec une variation des flux et des destinations selon les périodes.

Ces migrants incarnent, pour certains auteurs, un archétype de la « diaspora » (Lacoste, 1989) au sens que Gabriel Sheffer lui a conféré, à savoir une formation sociopolitique issue de la migration volontaire ou forcée de ses membres dans un ou plusieurs pays d'accueil. Par leur attachement à la «diaspora » libanaise, les individus exprimeraient leur sentiment d'appartenance à une société et à une culture d'origine. Par ailleurs, la diaspora étant un phénomène mondial, ses réseaux relieraient les multiples pays d'accueil et le pays d'origine (Sheffer, 2003).

La perspective macrosociologique, dominante dans ces travaux, conduit alors à mettre l'accent sur des pratiques de solidarité à l'œuvre (Abdulkarim, 1994 ; Abdelhady, 2006). Il est vrai que les migrants levantins du mont Liban ont joué un rôle fondamental dans la constitution de l'entité politique libanaise sous mandat français en 1920 (Lacoste, 1989). De même, les crises politiques et les guerres qu'a connues le Liban depuis ont souvent donné lieu à la mobilisation d'organisations de migrants. À la fin du conflit en $2006^{4}$, les ressortissants libanais à l'étranger se sont ainsi largement

chercheurs en sciences sociales après qu'ils ont subi des mesures de discrimination dans la majorité des pays où ils étaient implantés » (Rubbers, 2009 : 14-15). Car à partir des indépendances la littérature universitaire considère que ces communautés connaitront le même sort que la colonisation et disparaîtront. Par conséquent, les chercheurs s'intéressent plutôt aux entrepreneurs africains et à l'ouverture des marchés africains aux échanges mondiaux.

4 La guerre israélo-libanaise de 2006 éclata le 12 juillet pour se terminer le 14 août. Les forces se confrontant furent essentiellement l'armée israélienne et le Hezbollah, un parti politique armé participant au gouvernement libanais. L'armée libanaise a pris part, dans une moindre mesure, au conflit. Le Liban a subi, au cours de cette guerre, de lourdes pertes lors des at- 
mobilisés financièrement pour la reconstruction du pays (Hourani, 2007). Dans un même temps, l'approche en termes de "diaspora " conduit parfois à considérer le rapport des migrants libanais à leur terre d'origine comme immuable ainsi qu'à présupposer des logiques de solidarité. «Ils sont capables, à n'importe quel moment, de mobiliser les ressources ethniques, matérielles et morales, au service de la communauté » souligne ainsi Abdulkarim (1994, 218).

L'approche la plus répandue à propos des premières migrations libanaises en Afrique de l'Ouest consiste à corréler la migration et le contexte historique de la fin du XIX ${ }^{e}$ siècle en mettant l'accent sur les persécutions, les famines et les conflits armés organisés par l'Empire ottoman (Corm, 1989). Cette approche lisse une réalité plus complexe : les statistiques tirées des archives de l'administration de l'Afrique Occidentale Française (AOF) montrent que ces flux migratoires se produisaient en dehors des périodes de crises ; ce type de voyages, coûteux, écartait de fait les plus démunis et les plus sinistrés (Arsan, 2014: 26). Andrew Arsan considère que cette conception résulte d'une construction idéologico-politique qui s'origine dans les récits des intellectuels libanais du début du XXe siècle et fut ensuite nourrie par l'administration coloniale pour discréditer l'Empire ottoman. Si conflits et famines eurent une incidence sur la décision d'émigrer, la motivation première des migrants était, en effet, économique. Par ailleurs, ces flux migratoires ne sont pas homogènes. Ils se distinguent notamment par leurs origines géographiques. Les migrants sont principalement originaires du mont Liban et de la montagne du Sud-Liban, Jabal Amil, deux régions aux histoires économiques et sociales différentes. Alors que l'économie du mont Liban était orientée jusqu'au début du XXe siècle vers la sériciculture, le moteur économique du Sud-Liban était une agriculture plus traditionnelle et les échanges commerciaux avec les régions situées aujourd'hui au sud de la ligne de démarcation avec Israël. Les crises économiques et sociales ne frappèrent donc pas de la même manière et aux mêmes moments les deux montagnes (Arsan, 2014 : 45). Ces éléments invitent, plus largement, à discuter les approches prédominantes dans la littérature relative aux Libanais d'Afrique de l'Ouest.

\subsection{Les approches théoriques et les limites conceptuelles}

À partir des années 1930, le Sénégal s'impose comme foyer de concentration de Libanais en AOF. Nombre de migrants libanais participent au commerce arachidier. Ils jouent des rôles d'intermédiaires entre les grandes maisons de commerce euro-

taques israéliennes qui visaient notamment les infrastructures (aéroport, ponts, autoroutes, industries, etc.).

5 Le commerce arachidier connait une croissance exponentielle et offre des opportunités économiques aux migrants libanais jusqu'à la crise survenue dans les années 1960. 
péennes, telles que la Compagnie Française d'Afrique de l'Ouest d'une part, et les populations locales productrices d'arachides situées en milieu rural d'autre part. Ils furent de ce fait considérés comme des middleman minorities (Bonacich, 19736), à savoir des intermédiaires entre « [...] producteur et consommateur, employeur et employé, propriétaire et locataire, élite et masses ${ }^{7} »$ (Bonacich, 1973: 583).

Que ce soit dans la littérature sur les middleman minorities ou sur la "diaspora", l'absence de distinction entre primo-arrivants et descendants de migrants libanais tend à gommer la pluralité des origines sociales de ces migrations et les phases de l'histoire du Liban auxquelles elles correspondent.

Cette distinction s'avère toutefois nécessaire pour contextualiser les dissonances qu'évoquent nos enquêtés au sujet des rencontres entre primo-arrivants et descendants de migrants. Alors que ces derniers expriment en effet un besoin de se distancier des pratiques des «Libanais du Liban» parfois considérés comme "noninstruits » et "incultes », mus par de mauvaises intentions dans les affaires ou incapables de s'intégrer à leur environnement, les primo-migrants interrogés déplorent, quant à eux, l'éloignement des «Libanais d'Afrique » de leur terre d'origine et peuvent considérer leur proximité avec les locaux comme suspecte, allant jusqu'à les surnommer «nègres blancs ». Cette complexité des rapports internes à la «communauté » n'est guère mise en avant dans la littérature universitaire. Les «Libanais » y sont décrits comme des étrangers dans les sociétés d'accueil - ce qui s'inscrit dans une conception plus générale de l'homogénéité du groupe libanais en Afrique (Beuving, 2006 : 325). Dès 1975, O’Brien a pourtant pointé les limites de cette lecture et souligné l'hétérogénéité de la « communauté libanaise » en Afrique en insistant sur les stratifications internes, notamment socio-économiques, comme sur la concurrence à l'œuvre en son sein (O'Brien, 1975 : 97).

Des différences de statuts sociaux et économiques hiérarchisent en effet les rapports entre les membres de la minorité libanaise en Afrique de l'Ouest. Les origines géographiques partagées ne suffisent pas toujours à former une "communauté ", à savoir une construction symbolique reposant sur un entrelacs de facteurs sociaux, économiques et politiques (Cohen, 1985) : la communauté, loin d'être une structure figée, possède des frontières mouvantes et les conditions d'appartenance des individus qui la composent varient selon la perception de chacun.

La portée réductrice du concept de middleman minorities appliqué aux Libanais d'Afrique de l'Ouest a été soulignée par Souha Taraf-Najib (2000) et Andrew Arsan (2014 : 125). Le matériel collecté par Andrew Arsan dans les archives de l’AOF témoigne

6 Edna Bonanich englobe dans cette catégorie les Juifs d'Europe, les Chinois dans le sud-ouest de l'Asie, les Arméniens en Turquie et les «Syriens » en Afrique de l'Ouest (durant la première moitié du XXe siècle, les populations en provenance de la Syrie et du Liban étaient ainsi qualifiées).

7 «[... producer and consumer, employer and employee, owner and renter, elite and masses ». Traduit en français par nos soins. 
de la variété des rôles assumés par les acteurs économiques libanais dans la première moitié du siècle. À cette époque-là, les Libanais présents au Sénégal et en possession d'un capital économique et social, agissaient comme garants, auprès de l'administration coloniale, de leurs compatriotes candidats à la migration vers ce pays. Une hiérarchie du lien social s'établissait alors entre le «sponsor» et le nouvel arrivant. Les notables libanais pouvaient également agir sur la réorganisation des réseaux commerciaux en ville ou en milieu rural et se comporter en pourvoyeurs de crédits auprès des membres des réseaux.

La théorie des minorités intermédiaires tend également à occulter la complexité du marché en Afrique de l'Ouest et la diversité des populations urbaines. Elle relègue en arrière-plan le rôle joué par les autres groupes, locaux et européens, en tant qu'intermédiaires entre grandes maisons de commerce et petits producteurs locaux. Les multiples plaintes déposées par les petits commerçants européens auprès de l'administration coloniale pour dénoncer la pratique commerciale déloyale de ces migrants attestent pourtant de ce rôle (Arsan, 2014: 88). Les commerçants dioulas, auxquels les Libanais sont comparés dans les écrits administratifs, universitaires et journalistiques français de la première moitié du XXe siècle (Arsan, 2014: 96) ont également pu participer à ces médiations.

Pour conclure, les comportements économiques des «Libanais d'Afrique de l'Ouest » ne peuvent être réduits aux archétypes qui prédominent dans la littérature sur la « diaspora » ou les middleman minorities ${ }^{8}$. C'est d'autant plus le cas qu'une organisation économico-sociale complexe s'établit au Sénégal et à Dakar dans la première partie du XXe siècle, que les Libanais diversifient leurs activités économiques et montrent des signes d'installation à long terme alors qu'une partie d'entre eux est de nationalité sénégalaise, nous y reviendrons. En outre, les travaux sur les migrations africaines en Afrique de l'Ouest ne permettent pas de penser ensemble l'histoire de ces migrations et la réalité ouest-africaine contemporaine (Bourgi, 2005; Taan, 1988; Hanna, 1961).

\section{La mobilité régionale libanaise et la stratégie entrepreneuriale}

Dans l'espace subsaharien, des réseaux de commerce et de migration sont établis depuis l'époque précoloniale et connectent les villes - plaques tournantes du com-

\footnotetext{
8 Boris Adjemian signale les mêmes difficultés d'ordre théorique dans la littérature scientifique européenne en ce qui concerne la présence arménienne en Éthiopie: "Or pour décrire la faune indistincte des "Levantins», ce sont les figures de l'éternel marchand supposé être doué de la fibre du commerce, de l'intermédiaire parasite de l'entreprise coloniale, du profiteur sans scrupule concurrençant déloyalement les Européens en Afrique qui se sont imposées au début du XXe siècle » (Adjemian, 2012: 115).
} 
merce - de part et d'autre de la région (Walther, 2008). Sous la colonisation, avec l'avènement des États, les flux de marchandises et d'hommes exploitent les différentiels de part et d'autre des frontières étatiques. La mobilité des Libanais de Dakar, de Ouagadougou et d'Abidjan, s'inscrit dans ce système.

\subsection{La mobilité régionale et les conditions locales}

La somme des mobilités individuelles dessine en effet un "système " (Mabogunje, 1970) qui confère un sens relationnel aux espaces urbains: les uns en fonction des autres. La prise de décision relative à la mobilité repose sur des facteurs attractifs et répulsifs (Push et Pull), combinés à un calcul des coûts et des risques potentiels. L'accès aux données concernant la stabilité politique, les opportunités économiques, les obstacles administratifs et judiciaires ainsi que les caractéristiques du marché local s'avère ainsi être un élément décisif du projet migratoire. Ces données permettent au candidat à la migration de formuler une rationalité des avantages escomptés - une rationalité au demeurant tributaire de l'accessibilité et de la fiabilité de ces données.

La littérature sur les mobilités régionales à partir ou à destination des pays qui nous intéressent souligne que, dès les années 1960 et jusqu'aux débuts de la crise politique ivoirienne en $1999^{9}$, des flux de migrants originaires du Sénégal se dirigent vers la Côte d'Ivoire (Dumont, Kanté, 2010) ${ }^{10}$. Nos données montrent que ces flux incluent alors des Libanais du Sénégal ayant fait l'expérience des statuts de "migrants » et " d'étrangers » dans une société postcoloniale aspirant à se réapproprier des espaces sociaux, politiques et économiques (Mbodj, 1992; O’Brien, 1975). Au lendemain de l'indépendance du Sénégal, les Libanais sont en effet exclus en majorité de la nationalité sénégalaise ${ }^{11}$ - des décrets présidentiels permettent toutefois à certains d'entre eux d'y accéder.

Par ailleurs, les politiques de nationalisation des entreprises mises en œuvre à l'Indépendance ont contribué à limiter l'activité des Libanais dans le commerce ara-

\footnotetext{
9 En 1999, un coup d'État renverse le président Henri Konan Bédié. En avril 2000, Laurent Gbagbo est élu président. II gouverne sous tensions politiques jusqu'en 2010, date des élections présidentielles remportées par Alassane Ouattara. C'est alors le début d'affrontements armés, qui durent cinq mois et aboutiront à une guerre civile en 2011.

10 «Durant les quatre décennies qui suivent l'indépendance de 1960, ce pays, notamment grâce à sa stabilité politique, connaît l'essor du cacao, des cultures de plantations d'un dynamisme économique qui attirent des migrants de l'Afrique occidentale, donc y compris du Sénégal. Dans les années 1970, la sécheresse qui frappe les pays du Sahel ajoute un élément supplémentaire de repoussement et l'émigration s'y accroit » (Dumont, Kanté, 2010 : 72).

11 L'art. 5 de la loi sur l'acquisition de la nationalité précisait : "Est sénégalais l'enfant légitime né d'un père sénégalais. » La loi prévoyait en revanche une possibilité d'obtenir la nationalité par décret à laquelle pouvait s'opposer le gouvernement, notamment lorsque le demandeur possédait une autre nationalité (Loi n 61-10 du 7 mars 1961).
} 
chidier et de détail et les ont conduits, ainsi que leurs descendants, à se diriger, dès les années 1960, vers le commerce de gros et l'industrie, au Sénégal ou ailleurs.

L'assouplissement des politiques migratoires de la Côte d'Ivoire, tout particulièrement à l'égard des titulaires de passeports libanais, de même que la croissance économique impressionnante du pays (Michailof, 2005) ont pu conduire de nombreux «Libanais d'Afrique »-à savoir les personnes s'identifiant comme libanaises et vivant en Afrique, qu'elles y soient nées ou pas - à privilégier, jusqu'aux années 2000, cette destination. Le pays semble également attirer les candidats à la migration du Liban. La guerre civile libanaise qui éclate en $1975^{12}$ accentuera d'ailleurs cette tendance.

Les turbulences politiques ivoiriennes de la fin des années 1990 et du début des années 2000 ont toutefois modifié ce schéma migratoire: certains migrants prennent alors la direction du Liban, d'autres adoptent une stratégie de relocalisation dans la région. Une réflexion centrée sur les Libanais de Ouagadougou, capitale du Burkina Faso, peut permettre de penser plus précisément ces dynamiques. Depuis la crise ivoirienne, la présence des acteurs économiques libanais s'y est en effet accrue ${ }^{13}$. Bien que le Burkina Faso apparaisse économiquement moins attractif que d'autres pays de la région, sa stabilité politique ${ }^{14}$ a pu les attirer. Son taux de croissance, de l'ordre de six pour cent par an entre 1997 et 2006 (OCDE, 2008), s'est accompagné d'une augmentation des investissements étrangers ${ }^{15}$. Pour beaucoup d'investisseurs, cette dynamique économique a constitué une promesse de retour sur investissement à terme.

12 En 1975, une guerre civile éclate au Liban entre les différents partis qui constituent son paysage politique et confessionnel, des milices libanaises et des groupes armés palestiniens. Très vite, la Syrie fait intervenir son armée sur le territoire libanais. Les affrontements se poursuivent ensuite avec l'invasion israélienne en 1982 qui atteint la capitale Beyrouth. Le conflit ne prend fin officiellement qu'en 1990 avec l'établissement de l'accord Taëf.

13 Le consul honoraire du Liban au Burkina Faso estime lors d'un entretien que la population libanaise a ainsi doublé dans le pays, passant d'environ 600 à 1200 personnes entre 2000 et 2012. Aucun moyen n'est mis en œuvre par l'État libanais pour obtenir des statistiques fiables et nous sommes informés par les ambassades et le consulat honoraire qu'aucune personne non-membre du corps diplomatique n'a accès aux fichiers de l'ambassade. À l'ambassade, un fichier équivaut à une famille et les Libanais ne s'enregistrent pas systématiquement. Ces estimations sont donc établies à partir d'une projection (le nombre d'individus par fichier est multiplié par le nombre de fichiers présents à l'ambassade).

14 La tendance a basculé depuis Octobre 2014 lorsqu'un coup d'État a renversé le président Blaise Compaoré - au pouvoir durant 27 ans. Le Burkina Faso traverse depuis une période d'incertitude politique.

15 Selon les données de la Banque Mondiale, en 1992, le Burkina Faso enregistre des Investissements Direct Étrangers de l'ordre de 570000 dollars US. En 2000, les IDE dépassent les 23 millions de dollars américains (Banque mondiale, informations en ligne). 


\section{L'extraterritorialité des « Libanais du Sénégal »}

À partir de la fin des années 1990, Ouagadougou attire ainsi des flux de migrants et d'investissements ${ }^{16}$. Parmi ceux qui se sont installés et ont investi à Ouagadougou, figurent des «Libanais du Sénégal ». Cette mobilité prend deux formes.

La première correspond à une mobilité " par le bas $\|^{17}$ qui relève d'une initiative personnelle : l'individu s'appuie sur son propre réseau de connaissances pour construire son projet entrepreneurial et migratoire. La décision du potentiel migrant dépend alors de l'accès à des informations sur les différentes opportunités économiques du pays. Interrogé sur la manière dont il a obtenu des informations sur le marché ouagalais, un homme arrivé en 2000 explique :

Moi j'étais à Abidjan quand les problèmes ont commencé ${ }^{18}$ [...]. Je suis rentré en contact avec A. M., présent avec moi à Abidjan, que je connaissais depuis Conakry [en Guinée]. II a de la famille ici [à Ouagadougou]. II s'est donc renseigné auprès d'eux. Ils lui ont dit que le pays offrait des opportunités dans les affaires, qu'il y avait de la sécurité et qu'on pouvait y investir dans le commerce ${ }^{19}$.

Ce type de renseignements est véhiculé par des réseaux qui peuvent plus ou moins garantir leur fiabilité. Cet enquêté raconte avoir quand même " pris la peine de visiter et de vérifier l'état du marché » pour garantir l'information. Par ailleurs, cette décision inclut une analyse des coûts, notamment liés au déplacement.

La deuxième forme, que nous qualifions de mobilité "par le haut », correspond à une stratégie entrepreneuriale d'expansion. Les grandes entreprises fondées par des Libanais en Afrique ont participé activement à ce type de mobilité régionale. À titre d'exemple, on peut citer deux entreprises du Sénégal : Orca Déco ${ }^{20}$ et Batimat ${ }^{21}$. Les stratégies d'expansion régionale de ces deux groupes, visant à réduire les risques

16 Début 2000, les Burkinabè présents en Côte d'Ivoire fuient les perturbations politiques. Un grand nombre choisit de se réinstaller au Burkina Faso et d'investir dans des projets privés et des projets urbains publics (Bertoncello, 2010).

17 À plusieurs égards cette mobilité rappelle la «mondialisation par le bas » d'Alain Tarrius (2002). D'ailleurs, l'auteur considère que les Libanais d'Afrique ont participé dans l'élaboration du modèle économique auquel il fait référence. Mais l'informalité est une condition nécessaire que l'on ne retrouve pas dans notre enquête.

18 En référence au coup d'État de 1999 en Côte d'Ivoire

19 Entretien réalisé avec A. E. le 10/07/12 à Ouagadougou, Burkina Faso. Nous souhaitons garder l'anonymat de nos enquêtés.

20 Fondé en 2001 à Dakar, le groupe Orca Déco est présent aujourd'hui dans 15 pays africains (Groupe Orca Déco, En ligne). Leader dans la vente de mobiliers importés de Chine, les gérants ont fait le choix de la diversité à Abidjan annonçant en janvier 2016 l'ouverture d'un supermarché (Mieu, 2016).

21 Fondé en 1987 à Dakar, le groupe Batimat est présent aujourd'hui dans 11 pays africains (Groupe Batimat, En ligne). Ils se sont spécialisés dans la vente de matériaux de construction. Ils ont parallèlement investi dans l'industrie, implantant trois unités de transformation sidérurgique dans la région ouest-africaine. 
d'investissement dans certains territoires - en lien notamment avec les crises politiques et économiques - et à investir de nouveaux marchés, les ont conduits à adopter une logique d'étirement spatial des réseaux. Nous entendons par là l'extension des réseaux interpersonnels en dehors du territoire familier par le déplacement physique des personnes (Bensaâd, 2011). Ces deux groupes ont des succursales à Ouagadougou. Dans ces dernières, les postes clés sont occupés par des cadres « libanais » venus du Sénégal, choisis dans le réseau personnel des chefs d'entreprises et formés dans l'entreprise mère.

Le concept d'extraterritorialité permet de caractériser ces pratiques économiques et de mettre en exergue le rôle joué par les relations interpersonnelles et les logiques d'appartenance communautaires. À l'instar de ce qui a pu être observé dans le cadre des études sur les économies mafieuses, il faut souligner «le contrôle de l'origine géographique » des personnes recrutées pour garantir la confiance (Champeyrache, 2013 : 91). Ces aspects invitent à considérer le rôle joué par « la confiance » en matière d'accès à l'information préalable à l'investissement économique comme de gestion.

Les pratiques de mobilités par le haut et par le bas renseignent sur les motivations économiques à l'origine de la décision de s'installer à Ouagadougou. Mais dans quelles dynamiques sociales les Libanais venus du Sénégal, en possession d'une nationalité sénégalaise, s'inscrivent-ils à Ouagadougou ? Et comment se caractérisent leurs interactions avec les autres membres de la "communauté libanaise »? Les dixsept entretiens que nous avons conduits avec ces Libanais révèlent des liens continus entre ces deux espaces. Ils invitent à considérer l'expansion extraterritoriale de la vie sociale des Libanais de Dakar vers Ouagadougou.

Alors qu'ils dépendent des mêmes institutions diplomatiques et religieuses que les ressortissants libanais sur le sol burkinabé, les individus venus du Sénégal et en possession de la nationalité sénégalaise cultivent un fort sens d'appartenance au Sénégal et un fort besoin de se distancier de la manière « libanaise » d'être et de se comporter, envisagée parfois de manière dépréciative. Un ancien employé de la succursale d'Orca à Ouagadougou déclare ainsi : « Je ne veux pas crever à Ouagadougou. J’ai donné les consignes, je veux être enterré à Dakar, à côté de mon père. » Il poursuit en soulignant au sujet des «Libanais » qu' « ils ont beaucoup la folie des grandeurs et le m'as-tu-vu », autant de caractéristiques qui le poussent à prendre ses distances avec eux ${ }^{22}$. Un employé de la succursale Batimat refuse, quant à lui, d'être assimilé à un Libanais ou d'« être confondu avec ces villageois ${ }^{23}$ [qui ont] mauvaise réputation $»^{24}$.

22 Entretien réalisé avec R. H. le 19/05/2014 à Ouagadougou.

23 II se réfère ainsi aux Libanais qui viennent des zones rurales du Liban pour s'installer en Afrique. L'expression ainsi formulée a une connotation péjorative car elle évoque un manque de civilité à l'égard du pays d'accueil, de faibles qualifications ainsi que de mauvaises pratiques commerçantes.

24 Entretien réalisé avec R. N. le 30/12/2012 à Ouagadougou. 
La « communauté libanaise du Sénégal » est évoquée par plusieurs de nos interviewés et constitue une figure-clé de leurs récits de vie (Cohen, 1985). Au-delà du discours, son existence se matérialise sous différentes formes. Dans un premier temps, les liens familiaux et personnels maintiennent l'attachement au groupe. Les mariages et les obsèques au Sénégal sont des occasions parmi d'autres, pour ces migrants, de faire acte de présence et de réactiver les réseaux. Les stratégies matrimoniales peuvent également manifester leur volonté de renforcer ces liens, notamment lorsqu'une union se réalise entre une branche de la « communauté » à Ouagadougou et une autre à Dakar. La « communauté imaginaire et imaginée » (Anderson, 2006 [1983]) des Libanais du Sénégal que l'on retrouve dans la narrativité de leur «différence » se reflète dans les modes d'organisations économique et sociale.

\subsection{Les liens entre « nationalité " et « extraterritorialité »}

Plusieurs Libanais venus du Sénégal interviewés se différencient des autres «Libanais » en soulignant leur nationalité sénégalaise. Cet aspect, récurrent au cours des entretiens, invite à questionner la signification que revêt, pour eux, leur nationalité.

Les stratégies mises en œuvre par nos interviewés concernant l'acquisition des nationalités apparaissent d'ordre autant économique que social. La récurrence des conflits au Liban et la recherche d'opportunités économiques sont les deux explications qui prédominent lorsque la quête d'une nationalité différente est évoquée. Les nationalités les plus prisées sont alors les nationalités européennes ou nord-américaines, en raison notamment de la protection et des droits qu'elles ouvrent en termes de santé et d'éducation. Mais les nationalités ouest-africaines s'avèrent également avantageuses car elles offrent un accès privilégié aux activités économiques régionales. L'intégration régionale ouest-africaine facilite en effet le déplacement des individus, des marchandises et des capitaux (Kabbanji, 2011). Les Libanais du Sénégal, concernés par ces mobilités, ont cependant été longtemps exclus de la nationalité sénégalaise, la loi exigeant de prouver une ascendance paternelle sénégalaise. Cette législation a tout particulièrement pesé sur les médecins ${ }^{25}$. À la fin des années 1990, la modification de la loi a facilité l'accession à la nationalité sénégalaise ainsi que les procédures administratives afférentes.

L'obtention de cette nationalité permet l'ancrage de la population libanaise dans la vie économique et politique du Sénégal. Elle facilite certaines activités professionnelles, notamment les professions libérales et les activités politiques ${ }^{26}$. Elle est égale-

25 Comme l'explique l'un de nos interviewés, les Libanais ayant fait des études de médecine ne pouvaient exercer le métier que 10 ans après avoir obtenu la nationalité sénégalaise. Entretien réalisé avec A. T. le 17/02/2014 à Dakar, Sénégal.

26 De 2012 à 2014 un Libano-Sénégalais, Haïdar El-Ali, a occupé respectivement les postes de ministre de l'écologie et de la protection de la nature et ministre de la pêche et des affaires maritimes. 
ment indissociable des pratiques de mobilité de nos enquêtés, puisqu'elle facilite les entrées et les sorties du territoire sénégalais. Elle contribue également au développement de l'extraterritorialité des membres de la "communauté libanaise du Sénégal » en donnant «sens» à leur vie sociale. La nationalité concrétise à leurs yeux l'appartenance au lieu : « quand vous parlez de commerce des immigrants, on est... ma fille elle a des enfants, on est à la quatrième génération! On ne doit plus se considérer comme immigrants! On est quand même du pays, on a la nationalité sénégalaise ${ }^{27}$ ». Pourtant cette appartenance reste ambivalente (Leichtman, 2005) du fait des relations qu'entretiennent nos interviewés avec le reste de la population sénégalaise. On peut souligner, par exemple, que pour plusieurs d'entre eux, épouser un-e sénégalais-e qui ne soit pas d'origine libanaise paraît inconcevable. Les relations amicales paraissent également circonscrites par ce que nos interviewés nomment la "proximité culturelle » : la majorité des amis de nos interviewés sont des «Libanais comme eux ».

\section{Conclusion}

La présence libanaise en Afrique de l'Ouest a beaucoup changé au cours du XXe siècle, notamment en raison des reconfigurations locales et régionales, et les cadres théoriques mobilisés pour décrire et analyser cette présence ne permettent pas toujours de rendre compte de ces évolutions. Les concepts de « diaspora » et de middleman minorities, fréquemment utilisés dans les études sur les Libanais en Afrique, nécessitent ainsi d'être revisités à la lumière de nouvelles données de terrain. Les comportements entrepreneuriaux des Libanais, dont la mobilité régionale constitue une illustration, méritent également d'être intégrés dans une vision globale du marché africain. Les déplacements des individus dessinent, en effet, un système complexe liant des espaces urbains les uns aux autres. Plus spécifiquement, la mobilité reliant le Sénégal au Burkina Faso révèle l'extraterritorialité de la dynamique libanaise au Sénégal. L'acquisition des nationalités ouest-africaines facilite la mobilité régionale, elle sert également de support aux discours d'appartenance des individus - une appartenance au demeurant ambivalente et plurielle.

La recherche sur les Libanais en Afrique de l'Ouest profitera à l'avenir de l'avancée de la littérature universitaire sur les problématiques ethno-nationales (Bayart, Geschiere et Nyamnjoh, 2001) et sur les minorités en Afrique, parmi lesquelles figurent les minorités indiennes (Adam, 2010), arméniennes (Adjemian, 2012) et européennes (Rubbers, 2009). En effet, les recherches qui s'intéressent au continent de manière générale, et à la région ouest-africaine en particulier, éclairent les rouages d'une relation coloniale qui subsiste et qui régit les rapports entre citoyenneté, nationalité et « race » (Mbembe, 2010). Or, la présence de ces minorités en Afrique dépasse les simples dynamiques économiques et marchandes, telles que nous avons pu le voir

27 Entretien réalisé avec G. M. et N. M. le 04/02/2013 à Dakar, Sénégal. 
à propos des Libanais. Au cœur de forces sociales et politiques, ces derniers participent, bon gré mal gré, à la réinvention des "communautés imaginées " (Anderson, 2006 [1983]) des nations africaines. Portées par ces questionnements, les études sur les minorités en Afrique seront amenées à observer de plus près l'ancrage politique de ces populations, leur militantisme - anticolonial, entre autres - et les échanges de part et d'autres des limites communautaires.

\section{Bibliographie}

ABDELHADY D. (2006), « Beyond Home/Host Networks: Forms of Solidarity Among Lebanese Immigrants in a Global Era », Identities: Global Studies in Culture and Power, vol. 13, $\mathrm{n}^{\circ}$ 3, p. 427-453.

ABDULKARIM A. (1994), "La diaspora libanaise : une organisation communautaire», L'espace géographique, $\mathrm{n}^{\circ}$ 3, $\mathrm{p}$ 217-223.

ADAM M. (2010), « Les minorités d'origine indo-pakistanaise au Kenya, en Tanzanie et en Ouganda », Transcontinentales, $n^{\circ} 8 / 9$. En ligne, consulté le 24 mai 2016. URL : http://transcontinentales.revues.org/788.

ADJEMIAN B. (2012), «Les Arméniens en Éthiopie, une entorse à la "raison diasporique” ? Réflexion sur les concepts de diaspora marchande et de minorité intermédiaire », Revue européenne des migrations internationales, vol. 28, n³, p.107126.

AKYEAMPONG E. (2006), « Race, Identity and Citizenship in Black Africa: the Case of the Lebanese in Ghana », Africa, vol. 76, n 3, p. 297-323.

ANDERSON B. (2006 [1983]), Imagined Communities: Reflections on the Origins and Spread of Nationalism, London \& New York, Verso.

ARSAN A. (2014), Interlopers of Empire: The Lebanese Diaspora in Colonial French West Africa, London, C Hurst \& Co Publishers Ltd.

BANQUE MONDIALE, «Indicateurs (Burkina Faso) ». En ligne, consulté le 08 mai 2016. URL : http://data.worldbank.org/indicator/BX.KLT.DINV.CD.WD?page=2..

BAYART J.-F., GESCHIERE P., NYAMDJOH F. (2001), "Autochtonie, démocratie et citoyenneté en Afrique », Critique internationale, vol. 1, nº 10, p.177-194.

BENSAÂD A. (2011), «Aux marges du Maghreb, des tribus mondialisées: Réseaux commerciaux, réseaux tribaux et connexions politiques mondialisés depuis l'Ouest saharien », Méditerranée, n¹16. En ligne, consulté le 09 mai 2016. URL : http://mediterranee.revues.org/5349.

BERTONCELLO B. (2010), « Diaspora, développement et urbanisation : des Burkinabè de l'étranger acteurs des projets urbains de Zaca et Ouaga 2000 à Ouagadougou (Burkina Faso) », Annales de géographie, vol. 5, n 675, p. 560-583. 
BEUVING J. (2006), «Lebanese Traders in Cotonou: A Socio-cultural Analysis of Economic Mobility and Capital Accumulation ", Journal of the International African Institute, vol. 26, $\mathrm{n}^{\circ}$ 3, p. 324-351.

BINET J. (1975), «Les Libanais en Afrique francophone», Kroniek van Africa, vol. 3, n 6, p. $258-265$.

BONACICH E. (1973), "A Theory of Middleman Minorities », American Sociological Review, vol. 38, n 5 , p. 583-594.

BOURGI A. (2005), « Libanais en Afrique ou d’Afrique ?», Outre-Terre, vol. 2, nº 11, p. 149153.

CHAMPEYRACHE C. (2013), "L'économie mafieuse : entre principe de territorialité et extraterritorialité », Hérodote, vol. 4, n 151, p. 83-101.

COHEN A. (1985), Symbolic Construction of Community, London, Routledge.

CORM G. (1989), « La diaspora libanaise - Entretien avec Yves Lacoste », Hérodote, $n^{\circ}$ 53, p. $84-100$.

DESBORDES J. (1938), L’Immigration libano-syrienne en Afrique Occidentale Française, Poitiers, Renault.

DUMONT G.-F., KANTÉ S. (2010), «L'émigration sénégalaise, autant sud-sud que sudnord » in V. MORINIAUX et al. (dir.), Les mobilités, agrégation de géographie, Paris, SEDES, p. 69-88.

GROUPE BATIMAT, « Le Groupe Batimat en bref ». En ligne, consulté le 20 avril 2016. URL : http://www.groupebatimat.com/Le-Groupe-Batimat-en-bref.html?lang=fr.

GROUPE ORCA DECO, «Où sommes-nous ?». En ligne, consulté le 20 avril 2016. URL : http://www.orcatrend.com/ou-sommes-nous/.

HANNA M. (1961), "The Lebanese in West Africa », Al-Kulliyah, Middle East Forum, vol. XXXVII, $\mathrm{n}^{\circ} 1$, p. 32-37.

HOURANI G. (2007), «Lebanese Diaspora and Homeland Relations », Paper Prepared for the The Forced Migration and Refugees Studies Program, American University of Cairo, 23-25 October.

KABBANjI L. (2011), Politiques migratoires en Afrique de l'Ouest: Burkina Faso et Côte d'Ivoire, Paris, Karthala.

LACOSTE Y. (1989), «Éditorial : Géopolitique des diasporas », Hérodote, nº 53, p. 3-12.

LEICHTMAN M. A. (2005), « The Legacy of Transnational Lives: Beyond the First Generation of Lebanese in Senegal », Ethnic and racial studies, vol. 28, n 4, p. 663-686.

MABOGUNJE A. (1970), « Systems approach to a theory of rural-urban migration », Geographical Analysis, vol. 1, n 2, p. 1-18. 
MBEmbe A. (2010), Sortir de la grande nuit : essai sur l'Afrique décolonisée, Paris, La Découverte.

MBODJ M. (1992), « La crise trentenaire de l'économie arachidière » in M.-C. DIOP (dir.), Sénégal : Trajectoire d’un état, Dakar, CODESRIA, p. 95-135.

MiCHAILOF S. (2005), « Côte-d'Ivoire 2005 : bienvenue sur le Titanic !», Commentaire, vol. $2, \mathrm{n}^{\circ} 110, \mathrm{p} .393-403$.

MIEU B. (2016), « Côte d'Ivoire : centre commerciaux, gare à la concurrence ! », Jeune Afrique. En ligne, consulté le 20/04/2016.

URL : http://www.jeuneafrique.com/mag/289611/economie/cote-divoire-centrescommerciaux-gare-a-concurrence/.

O’BRIEN R. C. (1975), « Lebanese Entrepreneurs in Senegal: Economic Integration and the Politics of Protection », Cahiers d'études africaines, vol. 15, n 57, p. 95-115.

OCDE (2008), « Rapport perspectives économiques en Afrique, Burkina Faso ». En ligne, consulté le 19 avril 2016.

URL : http://www.oecd.org/fr/dev/emoa/40568810.pdf.

RUBBERS B. (2009), Faire fortune en Afrique : Anthropologie des derniers colons du Katanga, Paris, Karthala.

SERVICE PUBLIC, « Journal Officiel de la République du Sénégal, Loi nº 61-10 du 7 mars 1961 ». En ligne, consulté le 12 avril 2016.

URL : http://www.servicepublic.gouv.sn/assets/textes/T-nationalitesenegalaise.pdf.

SHEFFER G. (2003), Diaspora Politics, At Home Abroad, Cambridge, Cambridge University Press.

TAAN D. (1988), Les Libanais en Côte-d'Ivoire d'hier à aujourd'hui, Beyrouth, Allubnani.

TARAF-NAJIB S. (2000), "Immigration ancienne et territorialisation inachevée: les familles libanaises du Sénégal », Cahiers d'Études sur la Méditerranée Orientale et le monde Turco-Iranien, $\mathrm{n}^{\circ}$ 30, p. 273-295.

TARRIUS A. (2002), La mondialisation par le bas, les nouveaux nomades de l'économie souterraine, Paris, Balland.

WALTHER O. (2008), Affaires de patrons, villes et commerce transfrontalier au Sahel, Berne, Peter Lang. 